\title{
Longitudinal asymmetry in sunspot activity during the ascending and descending phase of the solar cycle
}

\author{
E. S. Vernova ${ }^{1}$, K. Mursula ${ }^{2}$, M. I. Tyasto ${ }^{1}$ and D. G. Baranov ${ }^{3}$ \\ ${ }^{1}$ Institute of Terrestrial Magnetism, Ionosphere and Radiowave Propagation of RAS, St. \\ Petersburg Filial, St. Petersburg, Russia, email: d.baranov@mail.ioffe.ru \\ ${ }^{2}$ University of Oulu, Oulu, Finland, email: Kalevi.Mursula@oulu.fi \\ ${ }^{3}$ A.F. Ioffe Physical-Technical Institute, St. Petersburg, Russia, email: d.baranov@mail.ioffe.ru
}

\begin{abstract}
The longitudinal distribution of sunspot activity for 1917-1995 is studied using vector sums of sunspot areas. It is shown that the sunspot distributions behave differently during the Ascending phase and Maximum (AM) of the solar cycle on one hand and for the Declining phase and Minimum (DM) on the other depicting maxima around roughly opposite longitudes. While the maximum of the distribution for the AM period is found around the Carrington longitude of $180^{\circ}$, the maximum for the DM period is at the longitude of about $0^{\circ}$.
\end{abstract}

The existence of active longitudes has been investigated by various techniques and for various indices of solar activity. (For a review see, e.g., Benevolenskaya et al. 2001 and reference therein). We consider the problem of the preferred solar longitudes on the base of sunspot data for 1917-1995 (Greenwich and Pulkovo observatories) using vector sums of sunspot areas. To evaluate quantitatively the longitudinal asymmetry of the sunspot distribution we have used the vector summing of the solar activity (Vernova et al. 2002). Each sunspot group is presented as a polar vector whose length equals the sunspot area and whose phase corresponds to the Carrington longitude of the group. Thus the $i$-th sunspot group on a day $k$ of a Bartels rotation $(k=1,2, \ldots 27)$ in question is presented as a polar vector $\vec{S}_{i k}$. A vector sum is calculated using all sunspot groups observed during each day of the Bartels rotation under consideration: $\vec{S}=\sum_{i, k} \vec{S}_{i k}$. Whereas the modulus of $\vec{S}$ can be considered as a measure of longitudinal asymmetry, the direction of the vector points to the Carrington longitude dominating during the given Bartels rotation. Calculating the vector sum of sunspots strongly reduces the stochastic, roughly symmetric sunspot activity and emphasizes therefore the more systematic and ordered part of the longitudinally asymmetric sunspot activity.

Long-term changes of the longitudinal asymmetry for North and South hemispheres were studied by plotting histograms of the vector $\vec{S}$ phase (Vernova et al. 2004). Two opposite types of the phase distribution were found for South hemisphere: while the histograms for AM period (Fig.1a) are convex (maximum close to $180^{\circ}$ of Carrington longitude), they are concave (Fig.1b) for the DM period (maximum close to $0 / 360^{\circ}$ longitude). Thus the observed shapes of the histograms imply that the two parts of the solar cycle have, on an average, roughly opposite phase distributions. Similar but slightly less systematic changes were found for the solar activity of the North hemisphere.

To stress the point of the alternate domination of the two active longitudes persisting for several solar cycles following procedure was used: data for the DM period were shifted by $180^{\circ}$. Then all data for the period 1917-1995 were combined in one histogram (Fig. 1c). The resulting histogram for the South hemisphere depicts a very systematic (almost 

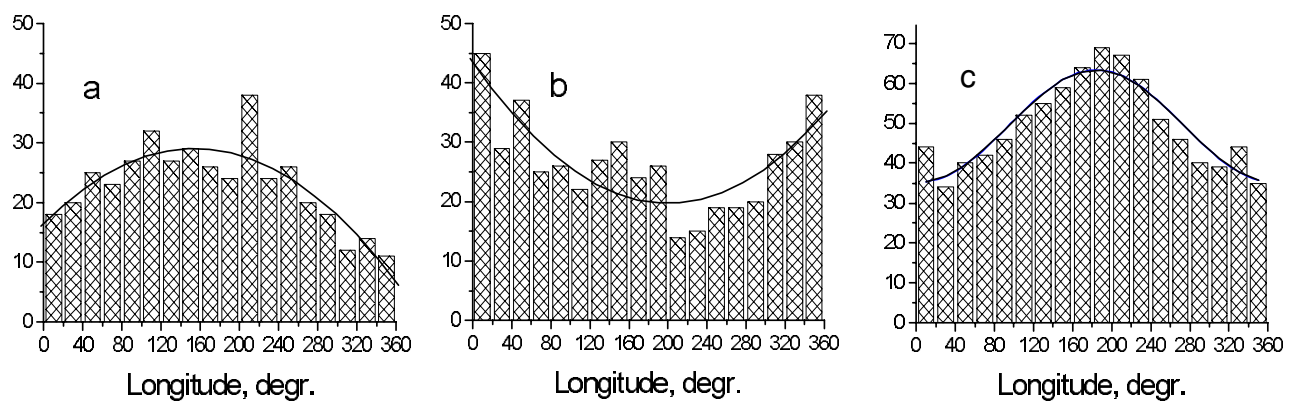

Figure 1. Longitudinal distribution of sunspots for the South hemisphere during 1917-1995. a) AM period. b) DM period. Curves indicate the best fitting second order polynomial. c) Phase distribution obtained by shifting the DM distribution by $180^{\circ}$ and summing with the AM distribution. Curve indicates the best fitting sinusoid.

Period Global magnetic field Polarity of the leading sunspot Active longitude, deg

AM of even cycle DM of even cycle AM of odd cycle DM of odd cycle

$\begin{array}{ccc}+ & + & 180 \\ - & + & 0 \\ - & - & 180 \\ + & - & 0\end{array}$

Table 1. Active longitudes and polarities of magnetic fields for the South hemisphere

sinusoidal) behavior of the phase with a pronounced maximum at about $180^{\circ}$. Note that Fig. 1c covers the data of the South hemisphere from the whole interval of 79 years under consideration. The maximum of the fitting sinusoid is located at $184 \pm 6^{\circ}$ longitude. The probability for the pattern to be due to random fluctuations is much less than $1 \%$.

A fundamentally new feature observed here is the fact that the average phase distribution in the AM period has a maximum at about $180^{\circ}$ of longitude, i.e., roughly $180^{\circ}$ contrary to the maximum of the DM period which is at about $0 / 360^{\circ}$. The times separating two characteristic periods are important intervals in the solar cycle. The time between solar maximum and the declining phase coincides with the inversion of Sun's global magnetic field. The time between the solar minimum and the ascending phase is related to the start of the new solar cycle and the change of the magnetic polarity of sunspots according to Hale's law. Polarity change of Sun's local and global magnetic fields for South hemisphere in the course of 22-year magnetic cycle is compared with the change of active longitude in Table 1. AM period corresponds to the epoch of coincidence of the global magnetic field polarity with the polarities of the leading sunspots. The change of the active longitude location by $180^{\circ}$ occurs in the DM period which corresponds to epoch with opposite signs of the global magnetic field and of the leading sunspots.

\section{References}

Benevolenskaya, E. E., Kosovichev, A. G., Scherrer, P. H. \& Lemen J. R. 2001 Synoptic Structure of the Solar Corona and Active Longitudes in EUV, http://soi.stanford.edu/ elena/.

Vernova, E. S., Mursula, K., Tyasto, M. I. \& Baranov, D.G. 2002 Solar Phys. 205(2), 371.

Vernova, E. S., Mursula, K., Tyasto, M. I. \& Baranov, D. G. 2004, Solar Phys. (in press). 